\title{
STOPP/START Criteria and Their Role as a Clinical Tool in Routine Practice
}

\author{
Denis O'Mahony \\ Department of Medicine (Geriatrics), University College Cork, Ireland.
}

Received: 23/08/2015

Accepted: 19/09/2015

Published: 24/09/2015

How to cite this article: O'Mahony D. STOPP/START criteria and their role as a clinical tool in routine practice. EJCRIM 2015;2:DOI: 10.12890/2015_ S1SP06

Conflicts of Interests: The authors declare that they have no conflicts of interest in this research.

Acknowledgements: This abstract has been presented as a lecture at $2^{\text {nd }}$ International Seminar REPOSI: Targeting the burden of polypharmacy in the elderly.

This article is licensed under a Commons Attribution Non-Commercial 4.0 License

Screening Tool of Older Persons' Prescriptions (STOPP)/Screening Tool to Alert to Right Treatment (START) criteria was first published in 2008, primarily as an alternative set of explicit criteria for potentially inappropriate medications (PIMs) to Beers criteria. A recent review in PubMed showed 114 articles on STOPP/START criteria including 5 systematic reviews, 9 reviews, 71 original research studies using STOPP/ START and 29 commentary articles from 31 countries in Europe, North America, Asia, Australia, South America, Middle East. Version 2 of STOPP/START criteria was published in 2014 following a Delphi panel assessment by 19 experts in Geriatric Medicine and Pharmacology from 13 European countries.

STOPP/START criteria are clinically relevant because they are associated with: (i) significant capture of ADEs in hospitalized older patients (unlike Beers 2003 criteria), (ii) significant improvement of medication appropriateness (RCT evidence), (iii) significant reduction of ADRs from use of STOPP in hospitalized older patients (RCT evidence), and (iv) significant reduction in median monthly medications costs in hospitalized older patients. Recent EU-funded projects (SENATOR and OPERAM) are now underway aimed at refining and testing by clinical trial software tools designed to rapidly deploy STOPP/START rules in multi-morbid elderly patients with a view to minimizing ADRs, healthcare costs and rehospitalisation. 